\title{
States of matter in massive planets
}

\author{
David J Stevenson $\dagger$ \\ Division of Geological and Planetary Sciences, California Institute of Technology, Pasadena, \\ CA 91125, USA
}

Received 17 August 1998

\begin{abstract}
This brief article addresses the question: among the very large number of interesting condensed matter physics issues, which are particularly interesting from a planetary perspective? Following some definitions and background, it is argued that we need to understand relevant firstorder phase transitions (especially the nature of the hydrogen phase diagram), the behaviour of the entropy (i.e., the Grüneisen parameter), the solubility and partitioning of minor elements (e.g. noble gases mixed with hydrogen), and microscopic transport properties, especially electrical and thermal conductivity. Examples are presented of how these issues influence current interpretations of the observations of Jupiter in particular. In the future, it may be possible to observe spectroscopically the compositions of extra-solar-system planets and brown dwarfs, and thereby learn more about the physics of these bodies.
\end{abstract}

\section{What is a massive planet?}

For the purposes of this discussion, a massive planet is a body that has been compressed to densities much higher than the normal (condensed) low-pressure state and yet is cold enough (i.e., degenerate) that there are no significant thermonuclear reactions. Roughly speaking, this places our consideration in the range $0.1 M_{\mathrm{J}}<M<80 M_{\mathrm{J}}$ where $M_{\mathrm{J}}$ is the mass of Jupiter ( 0.001 of the mass of the Sun). The upper bound of $80 M_{\mathrm{J}}$ is also the lower bound for stars on the main sequence, but it is common practice to think of bodies in the range of about 10 to $80 M_{\mathrm{J}}$ (so-called brown dwarfs; cf. Stevenson 1991) as forming like stars. These bodies also have a small but non-negligible deuterium-burning epoch. Accordingly, I will concentrate on bodies less massive than about $10 M_{\mathrm{J}}$.

The universe is mostly hydrogen and there are usually insufficient heavy elements around to make massive bodies as I have defined them out of material other than hydrogen. (The low-mass end of $0.1 M_{\mathrm{J}}$ does, however, approach Uranus/Neptune bodies for which water is a likely major constituent; cf. Stevenson 1982.) For example, it is widely believed that the discs (gaseous nebulae) from which planets form around stars are typically limited to of order $5 \%$ of a stellar mass, and only a few per cent of that is not hydrogen or helium; this limits the total available heavy elements to less than a Jupiter mass and this material is widely disseminated. Moreover, large assemblies of ice and rock are nuclei for the unstable infall and aggregation of enormous amounts of gas. Accordingly, massive planets will be almost invariably hydrogen rich and I will concentrate my attention on bodies of this composition.

† E-mail: djs@gps.caltech.edu.

0953-8984/98/4911227+08\$19.50 C 1998 IOP Publishing Ltd 
For such bodies, one can estimate the typical 'central' conditions to be as follows:

$$
\begin{aligned}
P_{c} & \sim 40\left(M / M_{\mathrm{J}}\right)^{2} \mathrm{mbar} \\
\rho_{c} & \sim 4\left(M / M_{\mathrm{J}}\right) \mathrm{g} \mathrm{cm}^{-3} \\
T_{c} & \sim 2 \times 10^{4}\left(M / M_{\mathrm{J}}\right) \mathrm{K} \text { (at } 4.5 \text { Ga since formation) }
\end{aligned}
$$

where the pressure and density come from the equation of hydrostatic equilibrium together with the equation of state of metallic hydrogen (dominated for most of the pressure range by the pressure of an ideal Fermi gas). 'Central' means outside of any possible ice-rock core which is small in mass but can have much higher pressures within it. These central cores are popular but uncertain attributes of giant-planet models (see Guillot et al (1997) for a recent discussion on this issue). The temperature is more uncertain but is estimated by equating the entropy of the deep interior to the entropy of the atmosphere at optical depth unity, as would be appropriate for a fully convective body. Of course, the entropy in the atmosphere depends on the radiating temperature of the planet, and this depends in turn on the heat content of the planet and the time for which it has existed. These bodies are believed to derive most of their luminosity from cooling from an initial hot state, and on this basis one can construct cooling curves and estimate current temperatures (Hubbard 1977, Stevenson 1982, Saumon et al 1992, Hubbard et al 1997).

Typically, the deep interior temperature is of order a few per cent of the Fermi temperature, but an order of magnitude or more in excess of the melting temperature of the classical one-component plasma. If we define a plasma parameter $\Gamma=e^{2} / a_{0} k T$ ( $e=$ electronic charge, $a_{0}=$ first Bohr radius, $k=$ Boltzmann's constant), then $\Gamma \sim 10$. This means that massive planets have dense Coulomb plasma interiors.

In our solar system, the bodies that qualify for my discussion are Jupiter and Saturn. However, there is rapidly growing evidence for massive planets in orbit around other stars. The field of extra-solar-system planets has rapidly evolved from a state of frequently retracted 'observations' to one where there is no longer any dispute about their existence. Although most of the current evidence comes from Doppler shifts measured for the primary (Mayor and Queloz 1995, Marcy and Butler 1996, Butler and Marcy 1996), future data will doubtless include spectra for the planets and there is already one example of a brown dwarf for which spectral information has been obtained (Oppenheimer et al 1995).

\section{The important questions}

At high pressure, there are many fascinating issues even if we confine ourselves to pure hydrogen. These include the rotational state of the molecules, relative populations of orthoand para-states, possibilities of superfluidity and superconductivity in the metallic phase and so on. However, many of these issues, fascinating though they may be, have no bearing on what takes place in giant planets because they concern temperatures far below those encountered in the planets. It is also possible to have an issue that is of theoretical interest at the temperature of interest (e.g., viscosity of the liquid) but of only limited (or less pressing) interest to the planetary modeller. So the list provided in table 1 is chosen with relevance in mind.

I now deal with each of these in turn. 
Table 1. The important issues.

\begin{tabular}{ll}
\hline Issue & Why it matters \\
\hline $\begin{array}{l}\text { First-order phase transition } \\
\text { (e.g. plasma phase transition or molecular } \\
\text { to metallic } \mathrm{H})\end{array}$ & $\begin{array}{l}\text { Affects density structure, heat transport, } \\
\text { thermal evolution, composition }\end{array}$ \\
\hline Entropy; $\gamma=(\partial \ln T / \partial \ln \rho)_{S}$ & Thermal state; existence of convection \\
\hline Solubility and partitioning & Thermal history; compositional state \\
\hline $\begin{array}{l}\text { Transport properties, especially electrical } \\
\text { and thermal conductivity }\end{array}$ & Thermal state; interpretation of magnetic field \\
\hline
\end{tabular}

\section{The importance of first-order phase transitions}

It has been proposed that hydrogen undergoes one or more first-order phase transitions as it converts from insulating molecules to the eventual hypothesized atomic, metallic, alkalimetal-like phase. Structural phase transitions have been documented for molecular hydrogen and it is widely believed that a band-overlap transition may well occur in the molecular state (though not necessarily with a density discontinuity that defines a first-order transition). At $T=0 \mathrm{~K}$, it seems very likely that the eventual yet elusive transition to atomic, metallic $\mathrm{H}$ should be first order but it is by no means clear whether this would continue to be first order at temperatures well above melting. At even quite high temperatures, it has been proposed that there might be a 'plasma phase transition' (PPT) whose nature might have as much in common with dissociation (of molecules into atoms) or ionization (atoms into protons and electrons) as with the usual picture of the molecular-metallic transition. Models of the PPT, both analytical and with computer simulation, find evidence for the PPT at conditions encountered in planets. Hubbard et al (1997) discuss this possibility and summarize current theoretical claims for the PPT. It should be stressed that the PPT is very difficult to pin down theoretically because it requires a very accurate computation of the energies of interactions among the various chemical 'species' $\left(\mathrm{H}_{2}, \mathrm{H}, \mathrm{H}^{+}, \mathrm{e}^{-}, \ldots\right)$. Since the hypothesized critical temperature (above which there is no first-order transition) is of order $10^{4} \mathrm{~K}$, corresponding to an energy of about $1 \mathrm{eV}$, any calculation must be made accurate to a small fraction of $1 \mathrm{eV}$, and this is a very daunting task. In Monte Carlo or molecular dynamics simulations, errors of this magnitude can easily arise if the system being modelled has many species (i.e., lacks natural repeatability as in a lattice or simple liquid).

It might be supposed that the importance of a first-order transition lies in the introduction of a density discontinuity within the planet. Surely this changes the bulk properties (radius as a function of mass, for example) in a major way? The answer is no, at least for density jumps of order $20 \%$ or less. The reason for this is that models without a firstorder phase transition inevitably must have some kind of interpolative equation of state that merges correctly with both the high-pressure and low-pressure limits (both of which are well known). As a consequence, it turns out that phase transitions have only very subtle effects on the radius-mass relationship. A phase transition could, of course, have a pronounced effect on seismology (the normal modes of the planet) and indeed much of what we know about the Earth's interior comes from the easily detected consequences of first-order phase transitions for seismic wave propagation. However, seismic data on massive planets are probably difficult to obtain because of the likely lack of strong excitation of these waves (unlike the case for the Sun, where the convective motions in the atmosphere have velocities 
that are a significant fraction of the speed of sound).

A first-order phase transition is more important for the thermal structure, including the thermal history (i.e., luminosity, a measurable property). The reason for this is that if you have a first-order phase transition, then there will be an entropy discontinuity at that phase transition (i.e., the convection will not penetrate the transition). This means that the entropy deep in the planet differs from that in the atmosphere, by an amount equal to the entropy of the phase transition. This can easily alter the estimate of central temperature by as much as a factor of two (Stevenson and Salpeter 1977).

A first-order phase transition is also important for partitioning. At the interface between two phases, Gibbs' phase rule applies:

$$
\mu_{i}(\mathrm{I})=\mu_{i}(\mathrm{II})
$$

where $\mu_{i}$ is the chemical potential of species $i$, and I, II refer to different phases (presumably of the dominant constituent hydrogen, within which $x$ is dissolved). Although this discontinuity is established by diffusion across the interface, convection will then accomplish the role of transporting that constituent up or down so as to communicate the consequences of the phase partitioning all the way to the atmosphere where it can be detected. Typically, a partition coefficient $x_{i}(\mathrm{I}) / x_{i}$ (II) differing from unity by a significant factor of order 2 is likely, where $x_{i}(\mathrm{I})$ is the mole fraction of species $i$ in phase I.

In section 7, I discuss the lack of evidence for a first-order phase transition in hydrogen inside Jupiter.

\section{Entropy and Grüneisen's parameter}

In a convective body, entropy is the parameter that characterizes temperature. Along an isentrope, the temperature varies with density in accordance with the value of the thermodynamic Grüneisen parameter $\gamma$ defined by

$$
\gamma \equiv(\mathrm{d} \ln T / \mathrm{d} \ln \rho)_{S} .
$$

Typical values believed appropriate for hydrogen are around 0.45 (the ideal-gas mixture of hydrogen and helium in Jupiter's atmosphere), $\sim 0.5$ (dense but insulating molecular hydrogen) and 0.6 (Coulomb plasma). However, it is well known that systems undergoing dissociation or ionization have small values because the entropy budget is being 'used up' to allow formation of a more disordered state rather than provide for higher temperature. Indeed, the procedure used by the Livermore group to analyse their conductivity measurements (Nellis et al 1995) predicts $\gamma<0$. For the pressure range of about 80 to $200 \mathrm{GPa}$, they predict that the isentrope declines slightly by about $100 \mathrm{~K}$ (at $T \sim 4000 \mathrm{~K}$ ) corresponding to $\gamma \sim-0.03$. An example of an everyday substance that has $\gamma<0$ is liquid water between $0{ }^{\circ} \mathrm{C}$ and $4{ }^{\circ} \mathrm{C}$. Of course, this small effect is quite likely to be overwhelmed by the positive contribution due to helium. Nonetheless, it is important to realize that the planet tells us that $\gamma>0$. The way that we know this comes from realizing (from Maxwell's relations) that $\gamma<0 \Leftrightarrow \alpha<0$, where $\alpha$ is the coefficient of thermal expansion. This follows from the identity

$$
\gamma \equiv \alpha K_{S} / \rho C_{P}
$$

where $K_{S}$ is the isentropic bulk modulus and $C_{P}$ is the constant-pressure specific heat, both of which must be positive for a stable thermodynamic system. Now if $\alpha<0$, then outward convection of heat is not possible (hot fluid elements have negative buoyancy) and heat cannot be removed; this would prevent us from explaining the heat flow out of these 
bodies. Even if $\gamma$ is positive but near zero, it has important consequences for our estimates of the temperature in that region.

\section{Solubility}

Consider a species $i$ that is dissolved in hydrogen. It has a chemical potential

$$
\mu_{i}=\mu_{i}^{0}+\Delta G_{i}+k T \ln x_{i}
$$

where $\mu_{i}^{0}$ is the chemical potential that this species would have if it were pure, $\Delta G_{i}$ is the Gibbs energy change that arises from placing those atoms in the hydrogen-dominated environment and $x_{i}$ is the mole fraction (assumed small) of the species. Clearly we can set $\mu_{i}=\mu_{i}^{0}$ to obtain the equilibrium (saturation) composition in hydrogen:

$$
x_{i}(\mathrm{sat})=\exp \left[-\Delta G_{i} / k T\right] .
$$

If this is larger than the cosmic abundance of an element, then that element will 'like' to form droplets and settle down deeper (since all candidate materials are more dense than hydrogen).

Table 2 lists the minimum $\Delta G_{i}$ for which insolubility is expected, assuming that $k T=0.7 \mathrm{eV}$ (a reasonable choice for megabar pressures).

\begin{tabular}{|c|c|}
\hline Element & $\begin{array}{l}\text { Minimum } \Delta G_{i} \\
\text { for insolubility } \\
(\mathrm{eV})\end{array}$ \\
\hline $\mathrm{He}$ & 2 \\
\hline $\mathrm{O}, \mathrm{C}$ & 7 to 8 \\
\hline $\mathrm{Ne}, \mathrm{N}$ & 9 \\
\hline $\mathrm{Mg}, \mathrm{Si}, \mathrm{Fe}$ & $\sim 12$ \\
\hline
\end{tabular}

Table 2. The minimum $\Delta G_{i}$ for which insolubility is expected.

The only plausible candidate is helium, both because it is very abundant but also because (like all noble gases) it is not very soluble in a metal. In the context of pseudopotential theory, it can be shown (Stevenson 1979) that the dominant contribution to $\Delta G_{i}$ for a noble gas is the energy cost arising from the need for the conduction electrons to orthogonalize to the core states of the noble-gas atom. (Helium and neon do not metallize until much higher pressures are reached.) Thus,

$$
\Delta G_{i} \sim n_{e}\left\langle\boldsymbol{k}\left|V_{p s}\right| \boldsymbol{k}\right\rangle
$$

where $n_{e}$ is the conduction electron density, $\langle\boldsymbol{k}|$ is a plane-wave state in the Fermi sea and $V_{p s}$ is the pseudopotential for the noble-gas atom in question (highly repulsive, of course). The matrix element is averaged over all $\boldsymbol{k}$-states in the Fermi sea. (In general, $V_{p s}$ is an operator, not just a function.)

Current estimates for $\Delta G_{\mathrm{He}}$ (or, roughly equivalently, for the critical temperature above which hydrogen and helium mix in all proportions) are quite uncertain (Stevenson 1975, 1979, Klepeis et al 1991, Pfaffenzeller et al 1995). It is not at all clear that more recent calculations are any more reliable than old estimates, since some of the recent work makes unrealistic assumptions about the physical ordering of the hydrogen and helium (e.g., assumes an ordered alloy). However, the estimates are such that limited solubility of helium in hydrogen is highly likely for Jupiter and Saturn. Strong evidence for this for Jupiter now exists, as discussed in section 7. 


\section{Transport properties}

Thermal conductivity is of interest in non-convecting regions, and there is reason to believe that the deep interiors may become conductive after a time of order several billion years has elapsed. This is a relatively minor correction to thermal histories even for the highest values that have been suggested (Stevenson and Ashcroft 1974).

Electrical conductivity is much more interesting. Of course, it is thought to be diagnostic of the metallic state in the shock-wave experiments, particularly those of the Livermore group that have recently attracted much attention (Nellis et al 1995). But regardless of whether you believe this to be an adequate diagnostic, these experiments are of great importance because they are directly applicable to the interpretation of giant-planet magnetic fields.

Although the exact criteria for dynamo generation are imperfectly known or understood (Stevenson 1983), the most important requirement is that the magnetic Reynolds number exceeds about ten:

$$
R e_{\mathrm{m}} \equiv v \ell / \lambda>10
$$

where $v$ is a characteristic fluid motion (presumably convective velocity), $\ell$ is a characteristic length scale associated with that motion and $\lambda$ is the magnetic diffusivity $\left(1 / \mu_{0} \sigma\right.$ in SI units, where $\sigma$ is the electrical conductivity). For plausible numbers $\left(v \sim 0.1\right.$ to $1 \mathrm{~cm} \mathrm{~s}^{-1}$, $\ell \sim 10000 \mathrm{~km})$ a sufficient magnetic Reynolds number is already attained at a resistivity of 0.01 to $0.1 \Omega \mathrm{cm}$, which the experiments suggest is attained at a pressure of 100-120 GPa, significantly less than the pressure of metallization. This can be tested by looking at the behaviour of the planetary field, as explained below.

\section{Jupiter}

Earlier missions to Jupiter have told us about the gravity and magnetic fields of the planet, and the most recent and ongoing Galileo mission has told us about atmospheric composition by direct in situ sampling. Models of Jupiter remain uncertain in some respects, even with new spacecraft data and the advances in our understanding of constituent materials, but they all have the following properties in common.

(1) A thin, outer region of ideal gas, predominantly hydrogen and helium, and adiabatic at least in the range of pressures between 1 bar and hundreds of bars, thus ensuring that the inside of the planet is hot. (When we look at Jupiter in the visible range, what we see are the ammonia cirrus clouds at somewhat less than one bar pressure.)

(2) A gradational transition into a dense fluid, without encountering any phase transitions. The planet has no surface. There may be a radiative zone (Guillot et al 1995) but the temperature reaches thousands of degrees rather quickly with radial distance down into the planet. At only $20 \%$ of the planet radius below the ammonia cirrus clouds $(80 \%$ in radius out from the planet centre) the pressure and temperature are already large enough to reach the conditions for which dynamo generation of the magnetic field is possible. This fits with the observed spectrum of Jupiter's field, which exhibits higher harmonics (quadrupole, octupole) consistent with this outer edge of the field-generating region (Stevenson 1983).

(3) Somewhat deeper down, perhaps at $70 \%$ of the radius, it is believed that helium has limited solubility in hydrogen. In accordance with equation (6), this arises because $\Delta G_{\mathrm{He}}$ increases more rapidly than $k T$, presumably because of the rapid increase in the number of conduction electrons. Helium raindrops form and settle to much deeper levels in the planet, where they probably dissolve because $\Delta G_{\mathrm{He}}$ no longer increases with pressure, yet $k T$ 
continues to increase. The existence of these raindrops is supported by the Galileo data for the atmosphere (Niemann et al 1996). The original idea for helium raindrops was provided by Salpeter (1973), modifying an earlier suggestion made by Smoluchowski (1967). If we define $Y$ as the mass fraction of helium in the atmosphere, then

$$
Y_{\mathrm{obs}}=0.236( \pm 0.004) \text {. }
$$

This should be compared with the value inferred from solar models for the 'primitive' (i.e., initial) abundance of helium within the Sun:

$$
Y_{\text {prim solar }}=0.272( \pm 0.005 ?)
$$

where the question-mark accompanying the error bar reflects the fact that this is not a direct observation but rather an inference from solar seismology, corrected for diffusion and possible mixing effects. Comparison of the two numbers suggests that Jupiter has undergone depletion by about $10-15 \%$. To be precise, the outer envelope (outer $\sim 30-40 \%$ by radius; perhaps only $10-20 \%$ by mass) has lost this fraction of its helium, which is now to be found deeper within the planet.

There is supporting evidence for this from the observation that neon is depleted in the Jovian atmosphere. This is a strong effect (the observed neon fraction is one tenth the solar mixing ratio) and thus not susceptible to concerns that we do not know the primordial value sufficiently well. Theory suggests that $\Delta G_{\mathrm{Ne}}$ is slightly larger than $\Delta G_{\mathrm{He}}$ (Czuchej et al 1989, Frongillo et al 1992, Roulston and Stevenson 1995, Stevenson 1996) which means, according to my previous discussion, that it cannot form its own droplets but will strongly partition into helium raindrops (more strongly than helium itself!)

Helium rain-out probably began about one billion years ago, triggered by the gradual cooling of the planet from an initial hot state, and the energy release from this process buffers the cooling (i.e., reduces the cooling rate, thus preventing still larger amounts of rain-out).

The existence of a first-order phase transition within Jupiter (the PPT for example) creates problems for interpretation for the planet: the most natural consequence of this phase transition would be to enhance the content of helium in the outer layers, contrary to what is observed.

\section{The future}

Progress in our understanding of these bodies requires more work on the properties of hydrogen and its mixtures with other elements. It is not necessary to map out entire binary or ternary phase diagrams; dilute (hydrogen-dominated) limits suffice. Here are the highest priorities, in ranked order.

(i) The nature of first-order phase transitions at megabar pressures and relevant temperatures $(>5000 \mathrm{~K})$. Does the PPT exist?

(ii) Solubility of helium in hydrogen under the same conditions and beyond.

(iii) Hydrogen-oxygen mixtures (the high-pressure extension of the low-pressure hydrogenwater system).

(iv) Hydrogen-neon, hydrogen-carbon and so on.

Aside from better theoretical and experimental input, there is the exciting prospect of learning more about these bodies by studying the spectra of planets around other stars. This can be expected to happen in the coming decade. 


\section{References}

Butler R P and Marcy G W 1996 Astrophys. J. 464 L153-6

Czuchej E, Rebentrost F, Stoll H and Preuss H 1989 Chem. Phys. 136 79-94

Frongillo Y, Plenkiewicz B, Plenkiewicz P and Jay-Gerin J-P 1992 Can J. Phys. 70 305-10

Guillot T, Chabrier G, Gautier D and Morel P 1995 Astrophys. J. 450 463-72

Guillot T, Gautier D and Hubbard W B 1997 Icarus 130 534-9

Hubbard W B 1977 Icarus 30 305-10

Hubbard W B, Burrows A, Saumon D, Marley M S and Freedman R S 1997 Phys. Plasmas 4 2011-5

Klepeis J E, Schafer K J, Barbee T W III and Ross M 1991 Science 254 986-9

Marcy G W and Butler R P 1996 Astrophys. J. 464 L147-51

Mayor M and Queloz D 1995 Nature 378 355-9

Nellis W J, Ross M and Holmes N C 1995 Science 269 1249-52

Niemann H B et al 1996 Science 272 846-9

Oppenheimer B R, Kulkarni S R, Matthews K and Nakajima T 1995 Science 270 1478-9

Pfaffenzeller O, Hohl D and Ballone P 1995 Phys. Rev. Lett. 74 2599-602

Roulston M S and Stevenson D J 1995 EOS AGU Fall Mtg (San Francisco, CA) abstract volume, p 343

Salpeter E E 1973 Astrophys. J. Lett. 181 L183-6

Saumon D, Hubbard W B, Chabrier G and Van Horn H M 1992 Astrophys. J. 391 827-31

Smoluchowski R 1967 Nature 215 691-5

Stevenson D J 1975 Phys. Rev. B 12 3999-4007

Stevenson D J 1979 J. Phys. F: Met. Phys. 9 791-801

Stevenson D J 1982 Annu. Rev. Earth Planet. Sci. 10 257-95

Stevenson D J 1983 Rep. Prog. Phys. 46 555-620

Stevenson D J 1991 Annu. Rev. Astron. Astrophys. 29 163-93

Stevenson D J 1996 unpublished

Stevenson D J and Ashcroft N W 1974 Phys. Rev. A 9 782-9

Stevenson D J and Salpeter E E 1977 Astrophys. J. Suppl. 35 221-37 